\title{
Evidence-based management of carotid artery disease
}

\author{
Sibu P Saha MD MBA, Thomas F Whayne Jr MD PhD, Debabrata Mukherjee MD
}

SP Saha, TF Whayne Jr, D Mukherjee. Evidence-based management of carotid artery disease. Int $\mathrm{J}$ Angiol 2010;19(1):e21-e24.

Stroke is a major cause of disability and mortality, and a major cause of stroke is carotid artery stenosis. This stenosis is caused by carotid atherosclerotic plaques, the prevention and management of which are the key to avoiding many resultant strokes. The plaque can either embolize to a cerebral artery or build up in a carotid artery, ultimately resulting in thrombosis and total occlusion. Noninvasive testing can now

$A$ n estimated 760,000 to 780,000 Americans experience a Astroke every year (1), ranking stroke as the third leading cause of death and the number one cause of disability among adults in the United States. Mortality following a stroke ranges from $20 \%$ to $30 \%$ initially and increases to approximately $50 \%$ by five years. Carotid artery stenosis (CAS) is believed to be responsible for approximately $20 \%$ to $30 \%$ of strokes. The most frequently found pathology in extracranial CAS is atherosclerosis. The management of CAS has significantly changed over the past 30 years, and currently both diagnosis and treatment are controversial (2). In the present article, we review the evidence for the current management of carotid atherosclerosis causing stenosis.

\section{PATHOLOGY}

Two basic mechanisms of stroke are hemorrhage and ischemia (3). Hemorrhagic stroke is often related to hypertension, while ischemic stroke is often related to atherosclerosis. In an ischemic stroke, atherosclerotic plaque from the heart or a carotid artery, especially from a bifurcation, degenerates and ruptures, resulting in the deposition of platelets and fibrin (Figure 1). Sometimes this plaque debris results in embolization to the distal cerebral circulation and causes a transient ischemic attack (TIA) or stroke. Alternatively, the plaque can build up and form a total thrombotic obstruction of the artery, thereby causing a significant decrease in the perfusion of blood to the brain.

\section{DIAGNOSIS}

A patient with cervical carotid artery disease may be completely asymptomatic or may present with symptoms of TIA, amaurosis fugax or a stroke. Physical findings may include decreased carotid pulses or a cervical bruit. However, a bruit may be present without significant disease in the carotid artery, and the absence of a bruit does not rule out carotid artery atherosclerosis. Clinical evaluation should be considered in patients who have risk factors for carotid artery disease, including hyperlipidemia, hypertension, diabetes, metabolic syndrome (MS), tobacco abuse and other predisposing factors. make the diagnosis of carotid stenosis. Medical management with plaque stabilization and platelet inhibition plays a key role in stroke prevention. Carotid endarterectomy and invasive carotid angioplasty stenting are also important for lesions with extensive progression, and patients with a very high overall risk may especially benefit from the latter procedure. A medical-surgical team approach is now greatly contributing to the avoidance of stroke and its devastation.

Key Words: Carotid artery disease; Endarterectomy; Stenting; Stroke

Diagnostic tests for extracranial CAS have changed. The conventional contrast angiogram, once considered to be the gold standard for diagnosis, is rarely used today. The current first line of evaluation is a duplex ultrasound examination. This is a combination of B-mode imaging and pulsed Doppler velocity spectrum analysis. Duplex examination is very reliable, painless, noninvasive and relatively inexpensive. Initially, cardiologists were reluctant to use a duplex examination as a definitive diagnostic test before any surgical intervention. However, years of experience have shown that complex carotid duplex ultrasound evaluation, along with quantitative Doppler spectral analysis, offers excellent imaging resolution to assess the presence and degree of flow restriction (4). One limitation of ultrasound study is the difficulty with differentiating total occlusion versus preocclusive disease or very tight stenosis of the internal carotid artery. Duplex ultrasound (Figure 2) has an added advantage of not only showing the lumen of the vessel but also characteristics of the plaque; this is often invaluable in deciding the appropriate form of treatment.

Alternative diagnostic tests for extracranial CAS include magnetic resonance (MR) angiography and computed tomography (CT) angiography. MR angiography is noninvasive and does not require an iodinated contrast medium. However, it overestimates the degree of stenosis, is inappropriate for people who are claustrophobic or unable to remain still on the examination table, and is often technically inadequate. MR angiography with gadolinium has been used in the past, but it is known that gadolinium is not innocuous; it can cause significant skin lesions, particularly in patients with abnormal kidney function, and may cause nephrogenic systemic fibrosis or nephrogenic fibrosing dermopathy (5). CT angiography is frequently used, often to delineate the extent of the disease including the intracranial component. However, CT angiography is expensive and has risks related to contrast media.

\section{MANAGEMENT}

Medical therapy to control classic risk factors The medical management of CAS focuses on decreasing individual cardiovascular (CV) risk factors as much as possible. 


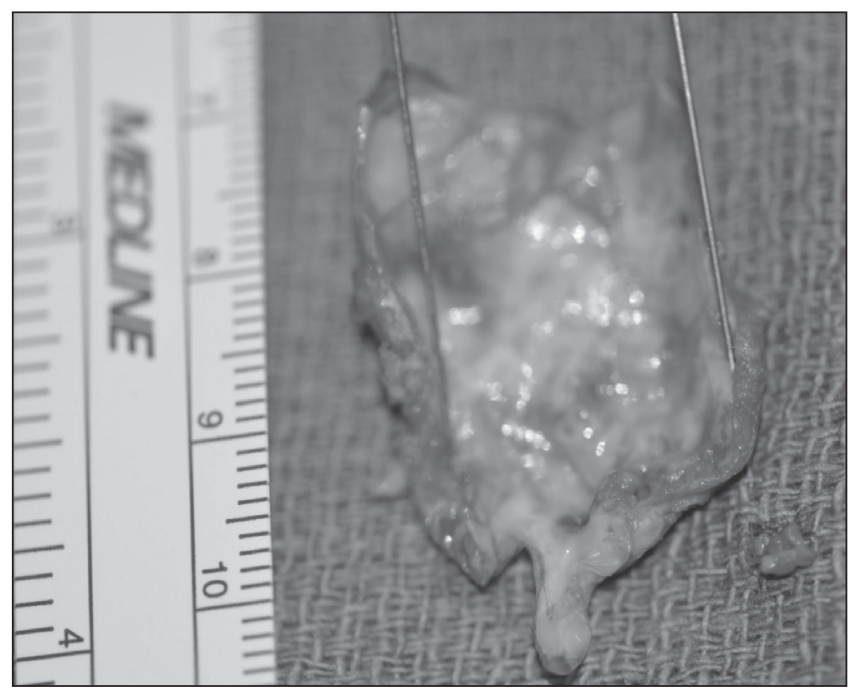

Figure 1) Ulcerative plaque surgically removed from a patient who presented with multiple episodes of transient ischemic attack

Low-density lipoprotein (LDL) is the most specific lipoprotein associated with coronary artery disease (CAD) (6); therefore, it can be considered the gold standard of CAD risk factors. In addition, only LDL has solid, well-accepted evidence that supports its reduction as beneficial in decreasing acute and chronic CV events (7) as well as associated diseases including CAS. Of all the medications available to manage and decrease LDL, statins are the cornerstone, and should be the first medication initiated after restricting cholesterol and saturated fat intake. For high-risk CV patients - including any patients with proven CAD, other peripheral vascular disease, CAD, diabetes mellitus (DM) or multiple CV risk factors - the goal for LDL is now established as less than $70 \mathrm{mg} / \mathrm{dL}$ (8).

The Pravastatin or Atorvastatin Evaluation and Infection Therapy - Thrombolysis In Myocardial Infarction 22 (PROVE IT-TIMI 22) substudy (9) showed that an even lower LDL level is better and that greatest clinical efficacy is achieved in acute coronary syndrome CAD patients who reach an LDL goal of less than $40 \mathrm{mg} / \mathrm{dL}$ or $40 \mathrm{mg} / \mathrm{dL}$ to $60 \mathrm{mg} / \mathrm{dL}$. Therefore, the 'lower is better' maxim for LDL appears to be well established, with no proven downside. On the other hand, the Effect of Ezetimibe Plus Simvastatin Versus Simvastatin Alone on Atherosclerosis in the Carotid Artery (ENHANCE) trial (5) studied the effect of ezetimibe plus simvastatin versus simvastatin alone on carotid intimamedia thickness (CIMT) and created much controversy. ENHANCE showed no significant difference in CIMT between the two groups and no significant difference in $\mathrm{CV}$ outcomes. However, the study was a short-term, small imaging study, using the highest risk group of CV patients, which consisted of heterozygous familial hypercholesterolemia patients, in whom it may be difficult to show a significant difference, especially over a short time period (10). Thus far, it can be considered that the study did nothing to negate the importance of maximum LDL lowering in high-risk CV patients, or the value of ezetimibe in achieving up to an additional $24 \%$ LDL reduction while keeping the statin at a safer, lower, long-term dose with the majority of statin benefit and pleiotropic effects (11).

Physicians and surgeons caring for patients with CAD, CAS, peripheral vascular disease, DM and/or MS are obligated to be aware of the mutually associated CV risk and to optimize

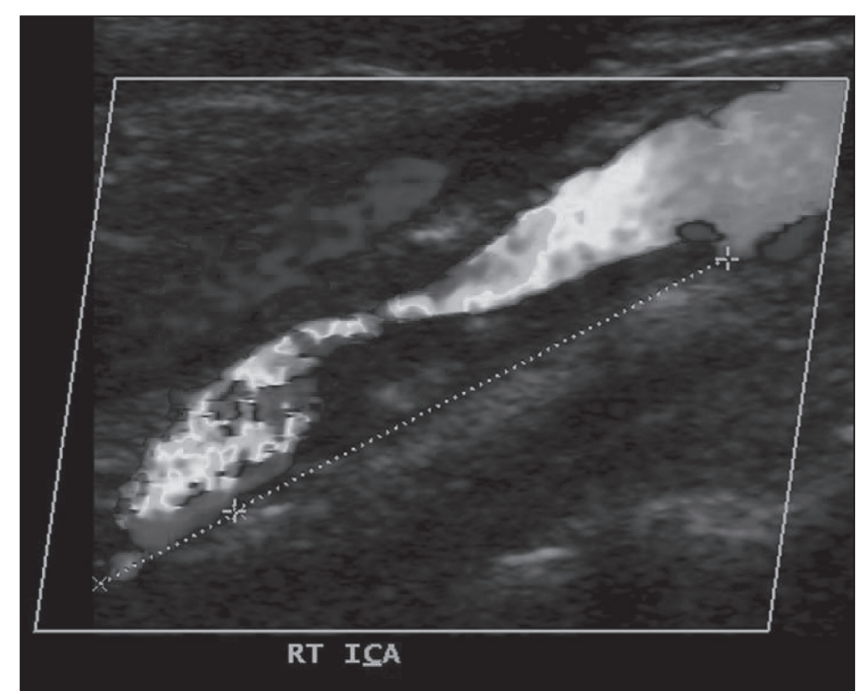

Figure 2) Ultrasound study showing severe stenosis of the right internal carotid artery (RT ICA)

the treatment of all CV risk factors. Although lowering LDL to below $70 \mathrm{mg} / \mathrm{dL}$ in DM patients with established high CV risk is indicated, close plasma glucose control of DM has only been marginally associated with decreased CV risk in the Action in Diabetes and Vascular Disease: Preterax and Diamicron Modified Release Controlled Evaluation (ADVANCE) trial (12). However, indications for extra-close control have not been clear cut, and have even been associated with problems, as shown in the Action to Control Cardiovascular Risk in Diabetes (ACCORD) trial (13). In the case of MS, all components should be treated aggressively as part of obligatory preventive management. The controversy over the definitions and etiology of MS as a clinical aggregate or collection of abnormalities in a population with bad health habits will continue (14-16). Nevertheless, efforts are underway to elucidate and understand the relationships. Maximum treatment of each component is warranted, as shown by the increase in CIMT related to each MS component added (17).

The Stroke Prevention by Aggressive Reduction in Cholesterol Levels (SPARCL) trial (18) was a randomized, double-blinded study designed to determine whether atorvastatin $80 \mathrm{mg} /$ day versus placebo would reduce the risk of fatal or nonfatal stroke in patients with no known coronary disease who had experienced a stroke or TIA within the previous six months. During a median follow-up of approximately five years, $11.2 \%$ of patients receiving atorvastatin and $13.1 \%$ of patients receiving placebo reached the primary end point of fatal or nonfatal stroke (five-year absolute reduction in risk, 2.2\%; adjusted HR, 0.84; 95\% CI 0.71 to 0.99; $\mathrm{P}=0.03$ ). The American Heart Association/American Stroke Association recommendations (19) state that on the basis of the SPARCL trial, administration of statin therapy with intensive lipidlowering effects is recommended for patients with atherosclerotic ischemic stroke or TIA, and without known CAD, to reduce the risk of stroke and $\mathrm{CV}$ events.

Therefore, there is good clinical trial evidence to support intensive CV risk factor management in patients with CAS. This is the standard of care whether in association with a nonobstructive CAS patient, or a pre- or postcarotid endarterectomy (CEA) or percutaneous intervention patient. 


\section{Medical therapy with platelet inhibitors}

Medical therapy has evolved from the simple use of acetylsalicylic acid to the management of risk factors and potential dual antiplatelet therapy. The efficacy of dual antiplatelet therapy with acetylsalicylic acid and clopidogrel was tested in the Management of Atherothrombosis With Clopidogrel in HighRisk Patients With Recent TIA or Ischemic Stroke (MATCH) trial (20). The MATCH trial did not show additional clinical value from adding acetylsalicylic acid $75 \mathrm{mg} /$ day to clopidogrel $75 \mathrm{mg} /$ day in high-risk patients with TIA or ischemic stroke over a period of 18 months. The European/Australian Stroke Prevention in Reversible Ischemia Trial (ESPRIT) (21) was a randomized, open-label study comparing acetylsalicylic acid $30 \mathrm{mg} /$ day to $325 \mathrm{mg} /$ day (median $75 \mathrm{mg} /$ day) with or without dipyridamole $200 \mathrm{mg} /$ day ( $83 \%$ of patients were placed on extended-release dipyridamole) in 2763 subjects with TIA, transient monocular blindness or minor stroke within six months of enrollment. The mean follow-up was 3.5 years. The risk for the primary outcome of death from all vascular causes, nonfatal stroke, nonfatal myocardial infarction or major bleeding complication was significantly lower in the dipyridamole plus acetylsalicylic acid arm (HR 0.80; 95\% CI 0.66 to 0.98) (21). The Prevention Regimen for Effectively Avoiding Second Strokes (PRoFESS) trial (22) demonstrated that the risks of recurrent stroke, or the composite of stroke, myocardial infarction or vascular death are similar with acetylsalicylic acid plus extendedrelease dipyridamole combination and clopidogrel monotherapy. The acetylsalicylic acid dose was $25 \mathrm{mg}$ twice daily, the dipyridamole dose was $200 \mathrm{mg}$ twice daily and the clopidogrel dose was $75 \mathrm{mg} /$ day, with a mean follow-up dosing period of 2.5 years.

The "Update to the American Heart Association/American Stroke Association recommendations for the prevention of stroke in patients with stroke and transient ischemic attack" (19) recommends that acetylsalicylic acid monotherapy ( $50 \mathrm{mg} /$ day to $325 \mathrm{mg} /$ day), the combination of acetylsalicylic acid and extended-release dipyridamole, and clopidogrel monotherapy are all acceptable options for initial therapy (class I, level of evidence A). Based on the ESPRIT trial, the combination of acetylsalicylic acid and extended-release dipyridamole is recommended over acetylsalicylic acid alone (class I, level of evidence B) (19).

\section{Surgery}

CEA was first performed in 1953. Since then, there has been a dramatic rise in this surgical procedure as a prophylaxis against stroke. In the 1980s, several reports implied that CEA had high complication rates in community hospitals. As a result, many neurologists challenged the utility of this procedure to prevent strokes, and some claimed that CEA was no better than medical treatment. In response to this concern, multiple randomized clinical trials were performed in the United States and Europe. Three high-quality studies, including the North American Symptomatic Carotid Artery Endarterectomy Trial (NASCET) $(23,24)$, European Carotid Surgery Trial (ECST) (25) and the Veterans Affairs (VA) Cooperative Study (26), showed a clear benefit of CEA in symptomatic patients when it was compared with medical therapy. NASCET and the ECST showed the efficacy of CEA in patients with symptoms of TIA or a nondisabling stroke; the RR reduction was $65 \%$ in the surgical group. These data were so impressive that the VA trial was stopped prematurely. Today, CEA remains the gold standard treatment in symptomatic patients with carotid artery disease unless they have serious comorbidities. In addition, three high-quality trials including the Asymptomatic Carotid Atherosclerosis Study (ACAS) (27), ECST and the VA Cooperative Study have shown benefits of CEA in asymptomatic patients. However, the benefit is lost if the incidence of perioperative stroke and/or death is more than 3\%. CEA should have, and in good programs does have, a low operative risk. Kang et al (28) reported on outcomes after CEA as part of the National Surgical Quality Improvement Program from the American College of Surgeons. Among 3949 CEA surgeries performed, the 30 -day stroke rate was $1.6 \%$, the death rate was $0.7 \%$ and the combined stroke/death rate was $2.2 \%$.

\section{Angioplasty and stenting}

Multiple studies have been and are currently being conducted to evaluate the place of carotid artery angioplasty and stenting. Both are accepted forms of treatment in patients who have a high operative risk. The superiority of stenting in asymptomatic and low-risk patients has not been shown at this time. Only a few randomized clinical trials have compared carotid stenting with CEA directly. The Carotid and Vertebral Artery Transluminal Angioplasty Study (CAVATAS) (29) randomly assigned 504 patients with low to moderate risk of stroke to either CEA or carotid angioplasty. Stroke (disabling or nondisabling) and death rates in both groups were nearly identical within 30 days. The Carotid Artery Stenting Versus Endarterectomy in High-Risk Patients (SAPPHIRE) trial (30) enrolled 334 patients between 2000 and 2002. The rates of primary end point (death, stroke or myocardial infarction at 30 days, plus ipsilateral stroke or death from neurological causes within 31 days to one year), based on intention-to-treat analysis, were $12.2 \%$ in the stent arm and $20.1 \%$ in the surgical arm $(\mathrm{P}=0.05)$. The Endarterectomy Versus Angioplasty in Patients With Severe Symptomatic Carotid Stenosis (EVA-3S) trial (31) was a randomized noninferiority trial comparing CEA with carotid stenting in symptomatic patients with $60 \%$ to $99 \%$ stenosis. This trial was terminated prematurely due to an increase in primary end points (any stroke or death within 30 days) in the stent arm (3.9\% versus $9.6 \%$, respectively). The Stent-Protected Angioplasty Versus Carotid Endarterectomy in Symptomatic Patients (SPACE) trial (32) was a noninferiority trial that enrolled 1200 patients to either carotid stenting with or without cerebral protection versus CEA. The primary end point of stroke or death was achieved in $6.84 \%$ in the stent arm versus $6.34 \%$ in the CEA arm. The recently reported International Carotid Stenting Study (ICSS) (33) showed superior results with surgery at 30 days postprocedure. The incidence of death, myocardial infarction and stroke was 5.1\% in the surgical arm and $8.5 \%$ with stenting.

Currently, given the proven efficacy and durability of CEA, surgical revascularization remains the standard of care in nonhigh-risk carotid stenosis. Carotid artery stenting needs to continue to prove its equivalence, if not superiority, and cost effectiveness when compared with CEA, before becoming the standard of care treatment for carotid stenosis. Presently, patients with symptomatic severe stenosis at high risk for surgery appear to be reasonable candidates for carotid stenting, and those not at high risk should be offered the choice to enroll 
in a randomized trial to objectively assess risks and benefits of stenting versus endarterectomy, or undergo CEA.

\section{CONCLUSIONS}

Stroke secondary to carotid atherosclerosis is preventable. Duplex scanning is the most valuable method in the diagnosis

\section{REFERENCES}

1. Leary MC, Saver JL. Annual incidence of first silent stroke in the United States: A preliminary estimate. Cerebrovasc Dis 2003;16:280-5.

2. Moore WS, Barnett HJ, Beebe HG, et al. Guidelines for carotid endarterectomy. A multidisciplinary consensus statement from the Ad Hoc Committee, American Heart Association. Stroke 1995;26:188-201.

3. Katzen I. Stroke. Cleveland Clinic Center for Continuing Education. <http://www.clevelandclinicmeded.com/medicalpubs/ diseasemanagement/neurology/ischemic-stroke/> (Accessed on December 29, 2009).

4. Bluth EI, Stavros AT, Marich KW, Wetzner SM, Aufrichtig D, Baker JD. Carotid duplex sonography: A multicenter recommendation for standardized imaging and Doppler criteria. Radiographics 1988;8:487-506.

5. Perreault P, Edelman MA, Baum RA, et al. MR angiography with gadofosveset trisodium for peripheral vascular disease: Phase II trial. Radiology 2003;229:811-20.

6. Sytkowski PA, Kannel WB, D'Agostino RB. Changes in risk factors and the decline in mortality from cardiovascular disease. The Framingham Heart Study. N Engl J Med 1990;322:1635-41.

7. Whayne TF. Evaluation critique of state of the art dyslipidemia management in general and with a special emphasis on the Indian population. Indian Heart J 2007;59:218-25.

8. Ballantyne CM. Cholesterol, lipids, and statins. Tex Heart Inst J 2005;32:378-9.

9. Wiviott SD, Cannon CP, Morrow DA, Ray KK, Pfeffer MA, Braunwald E. Can low-density lipoprotein be too low? The safety and efficacy of achieving very low low-density lipoprotein with intensive statin therapy: A PROVE IT-TIMI 22 substudy. J Am Coll Cardiol 2005;46:1411-6.

10. Whayne TF Jr. Is there a problem with ezetimibe or just ENHANCEd hype? Angiology 2008;59:661-3.

11. Mikhailidis DP, Sibbring GC, Ballantyne CM, Davies GM, Catapano AL. Meta-analysis of the cholesterol-lowering effect of ezetimibe added to ongoing statin therapy. Curr Med Res Opin 2007;23:2009-26.

12. Patel A, MacMahon S, Chalmers J, et al. Intensive blood glucose control and vascular outcomes in patients with type 2 diabetes. N Engl J Med 2008;358:2560-72.

13. Gerstein HC, Miller ME, Byington RP, et al. Effects of intensive glucose lowering in type 2 diabetes. N Engl J Med 2008;358:2545-59.

14. Takamiya T, Zaky WR, Edmundowics D, et al. World Health Organization-defined metabolic syndrome is a better predictor of coronary calcium than the adult treatment panel III criteria in American men aged 40-49 years. Diabetes Care 2004;27:2977-9.

15. Balkau B, Charles MA. Comment on the provisional report from the WHO consultation. European Group for the Study of Insulin Resistance (EGIR). Diabet Med 1999;16:442-3.

16. International Diabetes Federation. Metabolic syndrome: Consensus from the International Diabetes Federation could stop the cardiovascular disease time bomb. <http://www.idf.org/ metabolic-syndrome $>$ (Accessed on June 16, 2010).

17. Adolphe A, Cook LS, Huang X. A cross-sectional study of intimamedia thickness, ethnicity, metabolic syndrome, and cardiovascular risk in 2268 study participants. Mayo Clin Proc 2009;84:221-8. of carotid atherosclerosis. Statins can stabilize and even cause regression of carotid artery plaques. Antiplatelet therapy reduces microembolization. Acetylsalicylic acid offers the best results in symptomatic patients. Carotid artery stenting continues to improve and is often used for patients with high operative risks.
18. Amarenco P, Bogousslavsky J, Callahan A III, et al. High-dose atorvastatin after stroke or transient ischemic attack. N Engl J Med 2006;355:549-59.

19. Adams RJ, Albers G, Alberts MJ, et al. Update to the AHA/ASA recommendations for the prevention of stroke in patients with stroke and transient ischemic attack. Stroke 2008;39:1647-52.

20. Diener HC, Bogousslavsky J, Brass LM, et al. Aspirin and clopidogrel compared with clopidogrel alone after recent ischaemic stroke or transient ischaemic attack in high-risk patients (MATCH): Randomised, double-blind, placebo-controlled trial. Lancet 2004;364:331-7.

21. Halkes PH, van Gijn J, Kappelle LJ, Koudstaal PJ, Algra A. Aspirin plus dipyridamole versus aspirin alone after cerebral ischaemia of arterial origin (ESPRIT): Randomised controlled trial. Lancet 2006;367:1665-73.

22. Sacco RL, Diener HC, Yusuf S, et al. Aspirin and extended-release dipyridamole versus clopidogrel for recurrent stroke. N Engl J Med 2008;359:1238-51.

23. North American Symptomatic Carotid Endarterectomy Trial Collaborators. Beneficial effect of carotid endarterectomy in symptomatic patients with high-grade carotid stenosis. N Engl J Med 1991;325:445-53.

24. Barnett HJ, Taylor DW, Eliasziw M, et al. Benefit of carotid endarterectomy in patients with symptomatic moderate or severe stenosis. North American Symptomatic Carotid Endarterectomy Trial Collaborators. N Engl J Med 1998;339:1415-25.

25. Randomised trial of endarterectomy for recently symptomatic carotid stenosis: Final results of the MRC European Carotid Surgery Trial (ECST). Lancet 1998;351:1379-87.

26. Hobson RW II, Weiss DG, Fields WS, et al. Efficacy of carotid endarterectomy for asymptomatic carotid stenosis. The Veterans Affairs Cooperative Study Group. N Engl J Med 1993;328:221-7.

27. Endarterectomy for asymptomatic carotid artery stenosis. Executive Committee for the Asymptomatic Carotid Atherosclerosis Study. JAMA 1995;273:1421-8.

28. Kang JL, Chung TK, Lancaster RT, Lamuraglia GM, Conrad MF, Cambria RP. Outcomes after carotid endarterectomy: Is there a high-risk population? A National Surgical Quality Improvement Program report. J Vasc Surg 2009;49:331-8,339.e1.

29. Endovascular versus surgical treatment in patients with carotid stenosis in the Carotid and Vertebral Artery Transluminal Angioplasty Study (CAVATAS): A randomised trial. Lancet 2001;357:1729-37.

30. Yadav JS, Wholey MH, Kuntz RE, et al. Protected carotid-artery stenting versus endarterectomy in high-risk patients. N Engl J Med 2004;351:1493-501.

31. Mas JL, Chatellier G, Beyssen B, et al. Endarterectomy versus stenting in patients with symptomatic severe carotid stenosis. N Engl J Med 2006;355:1660-71.

32. Ringleb PA, Allenberg J, Bruckmann H, et al. 30 day results from the SPACE trial of stent-protected angioplasty versus carotid endarterectomy in symptomatic patients: A randomised non-inferiority trial. Lancet 2006;368:1239-47.

33. Brown M. International Carotid Stenting Study (ICSS). XVIII European Stroke Conference. Stockholm, May 26 to 29, 2009. 